\title{
Chapter 1 Static Digestion Models: General Introduction
}

\author{
Amparo Alegría, Guadalupe Garcia-Llatas, and Antonio Cilla
}

\begin{abstract}
Several in vitro methods have been developed to simulate the physiological conditions of the human gastrointestinal digestion, the simplest being the static methods. The following chapter clarifies the concepts of bioaccessibility and dialyzability, and describes the conditions ( $\mathrm{pH}$, enzymes, agitation, etc.) to be applied in oral, gastric and intestinal phases when assessing a food component (nutrient, bioactive or toxin) or a food product, in a single or multi-phase model. The advantages and disadvantages of the static models vs. dynamic and in vivo models are discussed, and a review of specific conditions applied on nutrients (minerals, vitamins, proteins, fatty acids, etc.) and bioactive compounds (carotenoids, plant sterols, etc.) from recent studies is provided. Currently, it must be considered that, although the static digestion conditions must be adapted according to the component or food sample to be studied, a harmonization and standardization of the models are needed in order to establish suitable correlations among in vitro and in vivo assays, as it has been defined for some food components (carotenoids, proteins and minerals).
\end{abstract}

Keywords Static models $\bullet$ Bioaccessibility $\bullet$ Dialyzability $\bullet$ Gastrointestinal $\bullet$ In vitro digestion

\subsection{Definition of Concepts: Bioavailability, Bioaccessibility and Bioactivity}

The term bioavailability can be defined as the fraction of ingested component available at the site of action for utilization in normal physiological functions, and is determined through in vivo assays (Guerra et al. 2012). Bioavailability is the result of three main steps: digestibility and solubility of the element in the gastrointestinal tract; absorption of the element by the intestinal cells and transport into the

\footnotetext{
A. Alegría • G. Garcia-Llatas • A. Cilla $(\bowtie)$

Nutrition and Food Science Area, Faculty of Pharmacy, University of Valencia,

Av. Vicente Andrés Estellés s/n, 46100 Burjassot, Valencia, Spain

e-mail: antonio.cilla@uv.es 
circulation; and incorporation from the circulation to the functional entity or target (Wienk et al. 1999; Etcheverry et al. 2012).

Bioavailability furthermore includes two additional terms: bioaccessibility and bioactivity. Bioaccessibility has been defined as the fraction of a compound that is released from its food matrix within the gastrointestinal tract and thus becomes available for intestinal absorption (typically established from in vitro procedures). It includes the sequence of events that take place during food digestion for transformation into potentially bioaccessible material but excludes absorption/assimilation through epithelial tissue and pre-systemic metabolism (both intestinal and hepatic). Bioactivity in turn includes events linked to how the nutrient or bioactive compound is transported and reaches the target tissue, how it interacts with biomolecules, the metabolism or biotransformation it may experience, and the generation of biomarkers and the physiological responses induced. Although bioavailability and bioaccessibility are often used indistinctly, it must be clarified that bioavailability includes bioactivity (Etcheverry et al. 2012).

\subsection{Static Methods}

\subsubsection{Solubility/Dialyzability}

In the past two to three decades, several in vitro methods have been developed to simulate the physiological conditions (temperature, agitation, $\mathrm{pH}$, enzyme and chemical composition) and the sequence of events that occur during digestion in the human gastrointestinal tract. Static methods (also called biochemical methods) are the simplest techniques in this respect and include two or three digestion steps (oral, gastric, and intestinal) whose products remain largely immobile in a single static bioreactor. These methods simulate a limited number of parameters of physiological digestion (to be described below), and do not mimic physical processes such as shearing, mixing, hydration, changes in conditions over time, or peristalsis (Fernández-García et al. 2009; Wickham et al. 2009).

In a first step, simulated gastrointestinal digestion is applied to homogenized foods or isolated compounds in a closed system, followed by determination of the amount of soluble compound present in the supernatant obtained by centrifugation or filtration (solubility methods). The amount of solubilized component can be used as a measure of the bioaccessibility of a nutrient or bioactive component. An important alternative methodological approach compared with previous systems is the introduction of a dialysis bag containing sodium bicarbonate, after gastric digestion of the food sample, and dialysis of soluble components across a semi-permeable membrane without removal of the dialyzed compounds. The use of a dialysis bag of a specific pore size also permits discrimination between high and low molecular weight components (Ekmekcioglu 2002; Etcheverry et al. 2012). 


\subsubsection{Digestion Conditions}

It is necessary to take into account in vivo conditions when applying in vitro digestion methods, in order to maximally reproduce them. In this sense, Ekmekcioglu (2002) summarized relevant aspects for bioavailability studies using in vitro models like peptic and pancreatic digestion (chemical and enzymatic composition of saliva, gastric juice, duodenal and bile juice, incubation time, temperature), adjustment of $\mathrm{pH}$, peristaltic frequency (shaking or agitation), osmolality, serosal composition, and permeability characteristics of the enterocyte monolayer, based on the physiological conditions.

The entire process in the mouth lasts from a few seconds to minutes, and since the salivary $\mathrm{pH}$ value is close to neutral, significant compound dissolution from food samples is not expected in this stage. This is why most methods only include the gastric and intestinal phases of digestion, and oral processing is perhaps the most difficult to simulate for solid foods. In place of such processing, use is normally made of a homogenization step, though this does not create a bolus. In the case of liquid foods or isolated food components, the homogenization and bolus formation phase is not performed, though salivary amylase may be added (Moreda-Piñeiro et al. 2011). For example, in studies on the hydrolysis of proteins there is no significant enzyme action in the mouth (Wickham et al. 2009), although an oral phase has been applied for other components (carotenoids, plant sterols and minerals). Some examples are shown in Table 1.1.

The gastric phase is performed with $\mathrm{HCl}$ or $\mathrm{HCl}$-pepsin under fixed $\mathrm{pH}$ and temperature conditions, for a set period of time. Food is homogenized in aqueous solution and typically pepsin is added following adjustment to $\mathrm{pH} 1-2$. The sample is then incubated at $37{ }^{\circ} \mathrm{C}$ during $1-3 \mathrm{~h}$, holding the $\mathrm{pH}$ constant. In the case of infant food the samples are acidified to $\mathrm{pH} 4$. A recent review has published a compilation of infant digestive conditions of gastric and duodenal phases with the aim of defining them for in vitro methods (Bourlieu et al. 2014).

Regarding the gastric enzymes, a minimum amount of 4,000-5,000 IU of pepsin seems to be necessary for optimal protein digestion (Ekmekcioglu 2002; Etcheverry et al. 2012). Wickham et al. (2009) reported that the pepsin digestion protocols that have been employed involve pepsin activities in the range of 8-12 units per mg of test protein, which may be considered far in excess of values likely to be found in the stomach. The protein dietary intake for an adult (around $75 \mathrm{~g}$ in $24 \mathrm{~h}$ ) would yield a ratio of $\sim 3 \mathrm{mg}$ protein/unit pepsin secreted, compared to $\sim 3 \mu \mathrm{g}$ protein/unit pepsin during digestion assays. Some authors add mucin in the gastric step in order to better simulate the physiological secretions. Gastric emptying times depend on meal composition, and in this regard meals with high fibre and fat contents can delay gastric emptying. Table 1.1 shows the conditions such as enzymes, $\mathrm{pH}$ and gastric emptying times recently used in some studies on different foods.

Intestinal digestion needs subsequent neutralization (usually with $\mathrm{NaOH}$ or $\mathrm{NaHCO}_{3}$ ), and incubation with pancreatic enzymes such as lipase, amylase, 







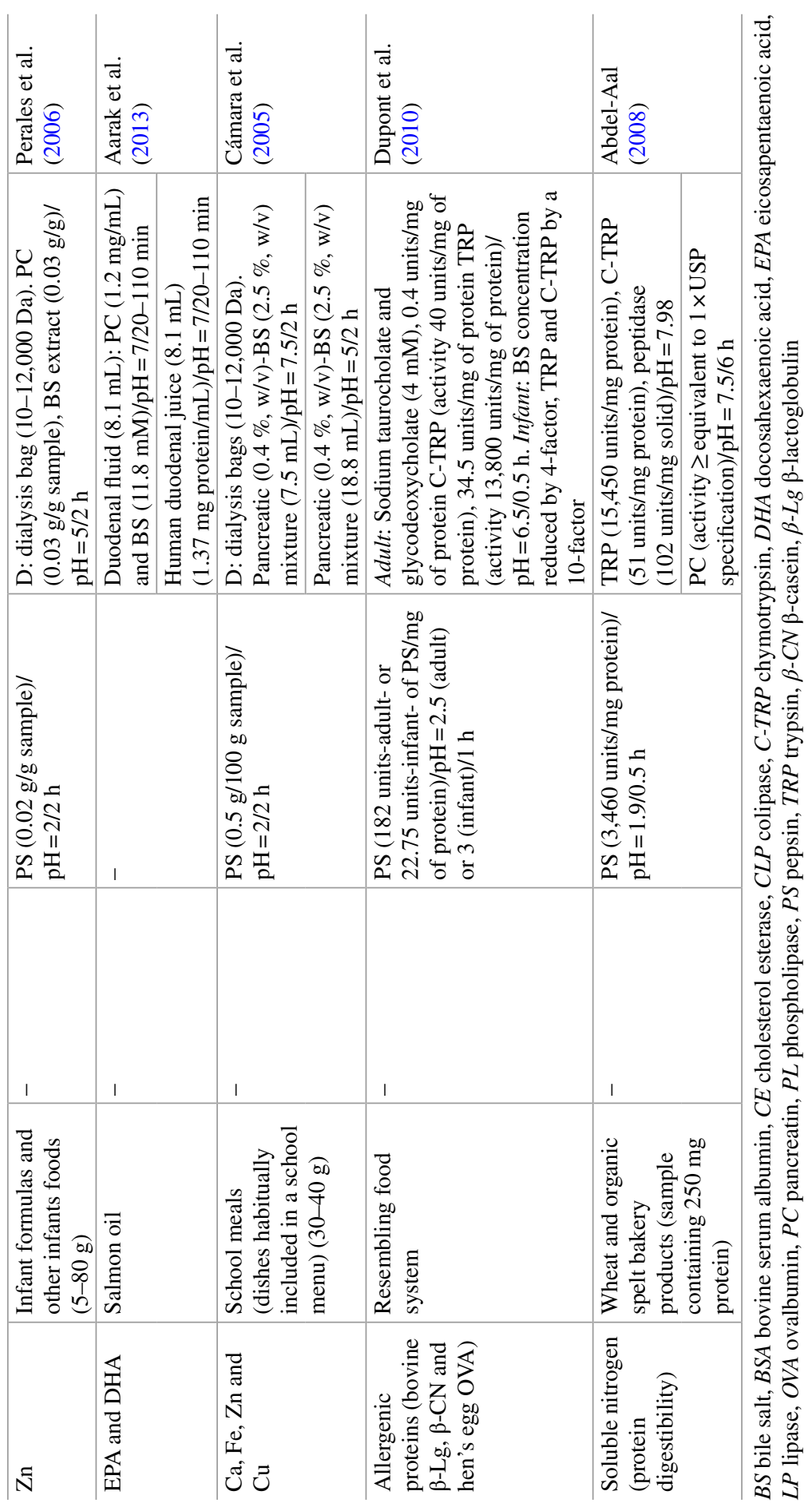


ribonuclease and protease with or without bile salts as emulsifiers (see Table 1.1). Since the majority of nutrients are absorbed in the jejunum ( $\mathrm{pH}$ 6.7-8.8) and ileum (6.8-7.7), most intestinal digestion studies adjust the $\mathrm{pH}$ to $6.5-7.5$ at $37{ }^{\circ} \mathrm{C}$ for 1-5 h (Ekmekcioglu 2002). Lipophilic compounds (carotenoids, plant sterols, etc.) partition into liposomes and micellar phases during intestinal digestion. Consequently, human pancreatic lipase and other specific enzymes (cholesterol esterase, phospholipase A2, co-lipase, etc.) are added to achieve more physiological conditions (Table 1.1). Other components such as phospholipids and calcium are also used in various in vitro models (Hur et al. 2011). Wickham et al. (2009) indicated that the colloidal phases should be included within the design of static digestion models used to assess the digestibility of protein allergens, because the multi-phase nature of the gastric and duodenal environments could play an important role in terms of allergenic protein potential, and thus in the conduction of risk assessments. These authors reviewed the studies on the role of physiological surfactants found in the gastric and the duodenal compartments in relation to potential allergens.

In studies that have used static methods, the choice of enzymes and incubation conditions is conditioned by the study objective. Thus, the application of such methods to a single nutrient has conditioned the use of a single enzyme, e.g., protein-pepsin, starch-amylase or lipid-lipase. Using a single purified enzyme offers the advantage of making standardization of the in vitro model easier; thereby allowing results to be obtained that are more reproducible among different laboratories. However, the digestion of a nutrient is influenced by other food components, and consequently the use of complex mixtures of enzymes affords results that more closely reflect the actual in vivo situation than the utilization of single purified enzymes. As an example, if protein digestion is carried out with three enzymes (trypsin, chymotrypsin and peptidase) in a single-step digestion process, greater protein digestibility (39-66\%) is obtained than in the case of a two-step digestion process with several enzymes (pepsin and pancreatic enzymes) (Abdel-Aal 2008; Hur et al. 2011). The enzymes are collected from human subjects, though a number of studies consider that it is possible to replace human pepsin, pancreatic lipase and co-lipase with porcine enzymes (Hur et al. 2011). Aarak et al. 2013 compared in vitro models using human and porcine intestinal enzymes applied to eicosapentaenoic acid (EPA) and docosahexaenoic acid (DHA) release from salmon, using only a duodenal digestion step. Results show that the human lipolytic enzyme system produces a comparatively higher release of EPA and DHA.

During peptic and pancreatic digestion, food samples are often incubated in a continuously shaking water-bath, although not all studies indicate the conditions used. A recently developed static device (Chen et al. 2011) allows agitation with a spherical probe, applying vertical movement within the vessel to create a flow pattern similar to that of the contraction waves of the stomach wall. 


\subsection{Applications: Advantages and Disadvantages}

Static models are particularly useful where there is limited digestion (e.g., gastric and/or intestinal steps), but are less applicable in total digestion studies, including colonic fermentation. These methods can be used to evaluate the influence of digestion conditions, and to carry out studies on the positive or negative effect of food structure (particle size, addition of emulsifiers, etc.), food composition (food fortification, etc.), dietetic factors (interactions between food components such as fibre, minerals, etc.) and food processing (thermal and non-thermal treatment, fermentation, etc.) upon nutrient and bioactive compound bioaccessibility, in order to establish the nutritional value of foods and improve food formulation/design. In conclusion, static models are predominantly used for digestion studies on simple foods and isolated or purified food components. Such studies not only contribute to improve food properties (nutritional or sensory) but also constitute preliminary trials producing evidence referred to possible nutrition and health claims, since it must be shown that the substance is digested and available to be used by the body (FernándezGarcía et al. 2009). An overview on different characteristics and conditions of the static models is represented in Fig. 1.1. A recent review assesses the importance of in vitro methods in nutritional, toxicological, pharmaceutical, and microbiological studies (Guerra et al. 2012).

Data from human intervention studies (in vivo assays) constitute the reference methods, whereas bioaccessibility studies (based on in vitro methods) are used as surrogates for predictive purposes. A number of disadvantages, such as limitations in experimental design, difficulties in data interpretation, high cost of equipment and labour, ethical constraints, inter-individual variations, and the lack of certified reference standards to compare data among studies limit the utility of in vivo methods (Fernández-García et al. 2009). In contrast, in vitro models are reproducible, since they allow better control of the experimental variables than animal or human studies, provided they are adequately validated and standardized, with the use of reference material if needed. In general, they are rapid and simple methods, since they only need materials that are routinely available in the laboratory, and are therefore relatively inexpensive and cost-effective. Furthermore, in vitro models allow a reduction of the sample size when this is a limiting factor. Static systems evaluate the aforementioned term "bioaccessibility", and can be used to establish trends in relative bioaccessibility, comparing the solubility of a component in different foods as a screening or categorizing tool. However, it is generally recognized that not all soluble or dialyzable compounds are absorbable.

Nevertheless, despite their potential and broad applicability, none of the static models reproduce the dynamic environment of the intestine. They cannot assess uptake or absorption, or transport kinetics, or measure nutrient or food component competition at the site of absorption as occurs in vivo. They are models lacking the complex mucosal barrier with all its regulatory processes, particularly hormonal and nervous control, feedback mechanisms, mucosal cell activity, complexity of peristaltic movements, gastric emptying or continuous changes in $\mathrm{pH}$ and secretion 


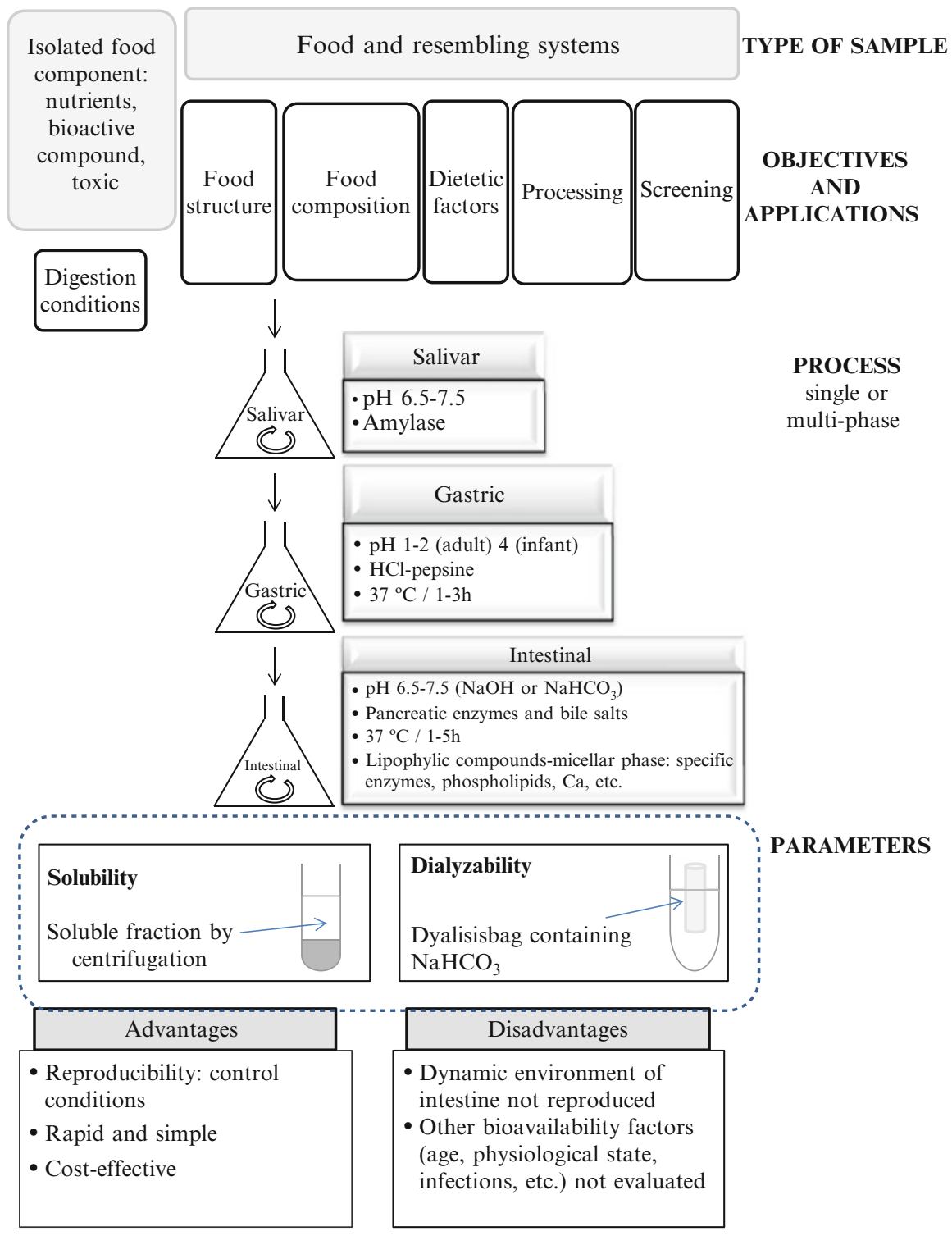

Fig. 1.1 Overview on static model conditions

flow rates, involvement of the local immune system, effects of the intestinal microflora and liver metabolism. Furthermore, factors that influence the bioavailability of a nutrient or bioactive compound, such as nutritional status, age, genotype, physiological state (e.g., pregnancy, lactation and obesity), or chronic and acute infections cannot be evaluated in static in vitro assays (see Fig. 1.1) (Etcheverry et al. 2012). 


\subsection{Static Versus In Vivo Digestion: Conclusions}

Few studies have evaluated the in vivo-in vitro correlation of results obtained in foods, and it is therefore difficult to properly assess the accuracy of the current in vitro assays. Some reviews, such as that published by Fernández-García et al. (2009), consider that a correlation effectively exists in the case of carotenoids, and that a measure of bioaccessibility might be sufficient as an estimation of how bioavailable a carotenoid is from the food in question (Etcheverry et al. 2012). Likewise, Butts et al. (2012), in reference to amino acid and protein digestibility, affirm that simple in vitro digestion methods have the potential to give useful measures of the in vivo behaviour. Van Campen and Glahn (1999) reviewed static solubility and dialyzability methods for estimating the availability of essential mineral elements, and found these methods to be in reasonable agreement with human absorption data, especially for iron. The authors also indicated that there has been much less development of in vitro methods for other trace minerals (zinc, copper, manganese, selenium) than for iron. A recent review (Etcheverry et al. 2012) compiles in vitro bioaccessibility and bioavailability methods applied to different nutrients, and recommends concrete methods for each nutrient. The need for more validation studies of in vivo-in vitro results is also discussed.

In conclusion, investigators who use static methods must consider how to adapt the static digestion conditions according to the composition of the sample and/or to food components under study — seeking a balance between technical simplification and accuracy, and always retaining the in vivo situation as reference with a view to maximally reproducing the physiological situation through the static model. In addition, it is necessary to know and assess the advantages and disadvantages of static in vitro digestion models for different food samples. Thus, there is urgent need for harmonization and standardization of the in vitro techniques, particularly the static methods. Furthermore, these methods must be validated with proper assessments of gastrointestinal human physiology, in order to afford improved study designs. In this context, although such methods are unable to reproduce all the conditions found in the in vivo setting, their validation at least will allow the comparison of preliminary results among laboratories, prior to the conduction of more advanced studies (dynamic in vitro studies, the use of cell cultures, or in vivo experimentation).

Open Access This chapter is distributed under the terms of the Creative Commons Attribution Noncommercial License, which permits any noncommercial use, distribution, and reproduction in any medium, provided the original author(s) and source are credited.

\section{References}

Aarak KE, Kirkhus B, Holm H et al (2013) Release of EPA and DHA from salmon oil - a comparison of in vitro digestion with human and porcine gastrointestinal enzymes. $\mathrm{Br} \mathrm{J}$ Nutr 110: $1402-1410$ 
Abdel-Aal ESM (2008) Effects of baking on protein digestibility of organic spelt products determined by two in vitro digestion methods. LWT 41:1282-1288

Alemany L, Cilla A, Garcia-Llatas G et al (2013) Effect of simulated gastrointestinal digestion on plant sterols and their oxides in enriched beverages. Food Res Int 52:1-7

Alminger M, Svelander C, Wellner A et al (2012) Applicability of in vitro models in predicting the in vivo bioavailability of lycopene and $\beta$-carotene from differently processed soups. Food Nutr Sci 3:477-489

Bourlieu C, Ménard O, Bouzerzour K et al (2014) Specificity of infant digestive conditions: some clues for developing relevant in vitro models. Crit Rev Food Sci Nutr 54:1427-1457

Butts CA, Monro JA, Moughan PJ (2012) In vitro determination of dietary protein and amino acid digestibility for humans. Br J Nutr 108:S282-S287

Cámara F, Amaro MA, Barberá R et al (2005) Bioaccessibility of minerals in school meals: comparison between dialysis and solubility methods. Food Chem 92:481-489

Chen J, Gaikwad V, Holmes M et al (2011) Development of a simple model device for in vitro gastric digestion investigation. Food Funct 2:174-182

Dupont D, Mandalari G, Molle D et al (2010) Comparative resistance of food proteins to adult and infant in vitro digestion models. Mol Nutr Food Res 54:767-780

Ekmekcioglu C (2002) A physiological approach for preparing and conducting intestinal bioavailability studies using experimental systems. Food Chem 76:225-230

Etcheverry P, Grusak MA, Fleige LE (2012) Application of in vitro bioaccessibility and bioavailability methods for calcium, carotenoids, folate, iron, magnesium, polyphenols, zinc, and vitamins B6, B12, D, and E. Front Physiol 3:1-21

Fernández-García E, Carvajal-Lérida I, Pérez-Gálvez A (2009) In vitro bioaccessibility assessment as a prediction tool of nutrient efficiency. Nutr Res 29:751-760

Granado-Lorencio F, Donoso-Navarro E, Sánchez-Siles LM et al (2011) Bioavailability of $\beta$-cryptoxanthin in the presence of phytosterols: in vitro and in vivo studies. J Agric Food Chem 59:11819-11824

Guerra A, Etienne-Mesmin L, Livrelli V et al (2012) Relevance and challenges in modeling human gastric and small intestinal digestion. Trends Biotechnol 30:591-600

Hur SJ, Lim BO, Decker EA et al (2011) In vitro human digestion models for food applications. Food Chem 125:1-12

Lacomba R, Salcedo J, Alegría A et al (2011) Effect of simulated gastrointestinal digestion on sialic acid and gangliosides present in human milk and infant formulas. J Agric Food Chem 59:5755-5762

Moreda-Piñeiro J, Moreda-Piñeiro A, Romarís-Hortas V et al (2011) In-vivo and in-vitro testing to assess the bioaccessibility and the bioavailability of selenium and mercury species in food samples. Trends Anal Chem 30:324-345

Peixoto RRA, Mazon EAM, Cadore S (2013) Estimation of the bioaccessibility of metallic elements in chocolate drink powder using an in vitro digestion method and spectrometric techniques. J Braz Chem Soc 24:884-890

Perales S, Barberá R, Lagarda MJ et al (2006) Bioavailability of zinc from infant foods by in vitro methods (solubility, dialyzability and uptake and transport by Caco-2 cells). J Sci Food Agric 86:971-978

Rodríguez-Roque MJ, Rojas-Grau MA, Elez-Martínez P et al (2013) Changes in vitamin C, phenolic, and carotenoid profiles throughout in vitro gastrointestinal digestion of a blended fruit juice. J Agric Food Chem 65:1859-1867

Van Campen DR, Glahn RP (1999) Micronutrient bioavailability techniques: accuracy, problems and limitations. Field Crop Res 60:93-113

Wickham M, Faulks R, Mills C (2009) In vitro digestion methods for assessing the effect of food structure on allergen breakdown. Mol Nutr Food Res 53:952-958

Wienk KJH, Marx JJM, Beynen AC (1999) The concept of iron bioavailability and its assessment. Eur J Nutr 38:51-75 